\title{
Generic transfer from GSp(4) to GL(4)
}

\author{
Mahdi Asgari and Freydoon Shahidi
}

\begin{abstract}
We establish the Langlands functoriality conjecture for the transfer from the generic spectrum of GSp(4) to GL(4) and give a criterion for the cuspidality of its image. We apply this to prove results toward the generalized Ramanujan conjecture for generic representations of $\operatorname{GSp}(4)$.
\end{abstract}

\section{Introduction}

Let $k$ be a number field and let $G$ denote the group $\operatorname{GSp}\left(4, \mathbb{A}_{k}\right)$. The (connected component of the) $L$-group of $G$ is $\operatorname{GSp}(4, \mathbb{C})$, which has a natural embedding into $\operatorname{GL}(4, \mathbb{C})$. Langlands functoriality predicts that associated to this embedding there should be a transfer of automorphic representations of $G$ to those of GL(4, $\mathbb{A}_{k}$ ) (see [Art04]). Langlands' theory of Eisenstein series reduces the proof of this to unitary cuspidal automorphic representations. We establish functoriality for the generic spectrum of $\operatorname{GSp}\left(4, \mathbb{A}_{k}\right)$. More precisely (cf. Theorem 2.4), we prove the following.

Let $\pi$ be a unitary cuspidal representation of $\operatorname{GSp}\left(4, \mathbb{A}_{k}\right)$, which we assume to be globally generic. Then $\pi$ has a unique transfer to an automorphic representation $\Pi$ of $\mathrm{GL}\left(4, \mathbb{A}_{k}\right)$. The transfer is generic (globally and locally) and satisfies $\omega_{\Pi}=\omega_{\pi}^{2}$ and $\Pi \simeq \widetilde{\Pi} \otimes \omega_{\pi}$. Here, $\omega_{\pi}$ and $\omega_{\Pi}$ denote the central characters of $\pi$ and $\Pi$, respectively.

Moreover, we give a cuspidality criterion for $\Pi$ and prove that, when $\Pi$ is not cuspidal, it is an isobaric sum of two unitary cuspidal representations of $\mathrm{GL}\left(2, \mathbb{A}_{k}\right)$ (cf. Proposition 2.2).

We give a number of applications of this result. The first is Theorem 3.3 which gives estimates toward the generalized Ramanujan conjecture for generic representations of the group GSp(4) (cf. $\S 3.1)$. We also prove in Theorem 3.7 that any generic unitary cuspidal representation of GSp $\left(4, \mathbb{A}_{k}\right)$ is weakly Ramanujan (cf. $\S 3.2$ for the definition). In $\S 3.3$ we use our main result to give an immediate proof that the spin $L$-function of a generic unitary cuspidal representation of $\operatorname{GSp}(4, \mathbb{A})$ is entire. This is due to the fact that the spin $L$-function of GSp(4) now becomes a standard $L$-function (or product of two such $L$-functions) of general linear groups. This fact has also been proved recently for a certain class of globally generic representations of $\operatorname{GSp}\left(4, \mathbb{A}_{k}\right)$ by Takloo-Bighash using different methods from ours.

We should note here that the transfer from GSp(4) to GL(4) has been expected by experts in the field for a long time. It is our understanding that Jacquet, Piatetski-Shapiro and Shalika knew how to prove this result, at least in principle, but, as far as we know, their result was never published. We should also point out that their proof is based on methods that are fairly disjoint from ours.

Our method of proof is to start with our earlier, more general but weaker, result on generic transfer from GSpin groups to GL (cf. [AS06]). This gives us the existence of $\Pi$. We then use the

Received 5 January 2005, accepted in final form 15 August 2005.

2000 Mathematics Subject Classification 11F70, 11R42, 22E55.

Keywords: Langlands functoriality, automorphic representation, $L$-function.

First author partially supported by the NSF grant DMS-0111298 at IAS; second author partially supported by the NSF grant DMS-0200325.

This journal is (C) Foundation Compositio Mathematica 2006. 


\section{Asgari And F. Shahidi}

results of Piatetski-Shapiro and Soudry on analytic properties of $L$-functions of GSp(4) twisted by GL(1) and GL(2) to get more information about the representation $\Pi$. It is exactly the lack of such results in the general case of GSpin groups that prevents us from carrying out our analysis for the more general case for now. However, as we pointed out in [AS06], there are currently two ways to overcome this problem. One is to prove an analogue of descent theory for these groups as was done for classical groups by Ginzburg, Rallis and Soudry [GRS01, Sou05]. The other is to use techniques along the lines of [Kim01, Kim02].

The case of the transfer for all automorphic representations of GSp(4) (whether generic or not) requires Arthur's trace formula. The process and the expected issues in this case are outlined in [Art04].

\section{Main result}

Let $k$ be a number field and let $\mathbb{A}=\mathbb{A}_{k}$ denote its ring of adeles. We define the similitude symplectic group of degree four via

$$
\operatorname{GSp}(4)=\left\{g \in \mathrm{GL}(4):{ }^{t} g J g=\mu(g) J\right\},
$$

where

$$
J=\left(\begin{array}{cccc} 
& & & 1 \\
& & 1 & \\
-1 & & &
\end{array}\right)
$$

and $\mu(g) \in \mathrm{GL}(1)$ is the similitude character. We fix the following parametrization of the elements of the maximal torus $\mathbf{T}$ in $\operatorname{GSp}(4)$ :

$$
\mathbf{T}=\left\{t=t\left(a_{0}, a_{1}, a_{2}\right)=\left(\begin{array}{llll}
a_{0} a_{1} a_{2} & & & \\
& a_{0} a_{1} & & \\
& & a_{0} a_{2} & \\
& & & a_{0}
\end{array}\right)\right\} .
$$

The above agrees with our previous more general notation for the group GSpin $(2 n+1)$ in [AS06]. Recall that the group GSp(4) is identified with GSpin(5).

Let $\pi=\bigotimes_{v} \pi_{v}$ be a globally $\psi$-generic unitary cuspidal automorphic representation of GSp $(4, \mathbb{A})$. Here, $\psi=\bigotimes_{v} \psi_{v}$ is a non-trivial additive character of $k \backslash \mathbb{A}$ defining a character of the unipotent radical of the standard upper-triangular Borel subgroup in the usual way. We fix $\psi$ now and do not repeat it in the rest of this paper. Let $S$ be any non-empty finite set of non-archimedean places $v$, which includes those $v$ with $\pi_{v}$ or $\psi_{v}$ ramified. We proved in [AS06] that there exists an automorphic representation $\Pi=\bigotimes_{v} \Pi_{v}$ of $\mathrm{GL}(4, \mathbb{A})$ such that $\Pi_{v}$ is a local transfer of $\pi_{v}$ for $v$ outside of $S$.

To be more explicit, assume that $v \notin S$. If $v$ is archimedean, then $\pi_{v}$ is given by a parameter $\phi_{v}: W_{v} \longrightarrow \operatorname{GSp}(4, \mathbb{C})$, where $W_{v}$ is the Weil group of $k_{v}$ (cf. [Lan89]). Let $\Phi_{v}: W_{v} \longrightarrow \operatorname{GL}(4, \mathbb{A})$ be given by $\Phi_{v}=\iota \circ \phi_{v}$, where $\iota: \operatorname{GSp}(4, \mathbb{C}) \longrightarrow \mathrm{GL}(4, \mathbb{C})$ is the natural embedding. Then $\Phi_{v}$ is the parameter of $\Pi_{v}$.

If $v \notin S$ is non-archimedean, then $\pi_{v}$ is the unique unramified subquotient of the representation induced from an unramified character $\chi$ of $\mathbf{T}\left(k_{v}\right)$ to $\operatorname{GSp}\left(4, k_{v}\right)$. Writing

$$
\chi\left(t\left(a_{0}, a_{1}, a_{2}\right)\right)=\chi_{0}\left(a_{0}\right) \chi_{1}\left(a_{1}\right) \chi_{2}\left(a_{2}\right),
$$

where $\chi_{i}$ are unramified characters of $k_{v}^{\times}$and $a_{i} \in k_{v}^{\times}$, the representation $\Pi_{v}$ is then the unique irreducible unramified subquotient of the representation of $\operatorname{GL}\left(4, k_{v}\right)$ parabolically induced from the 


\section{Generic transfer From GSp(4) TO GL(4)}

character

$$
\chi_{1} \otimes \chi_{2} \otimes \chi_{2}^{-1} \chi_{0} \otimes \chi_{1}^{-1} \chi_{0}
$$

of $\mathbf{T}\left(k_{v}\right)$.

Moreover, we proved that $\omega_{\Pi}=\omega^{2}$, where $\omega=\omega_{\pi}$ and $\omega_{\Pi}$ denote the central characters of $\pi$ and $\Pi$, respectively, and for $v \notin S$ we have $\Pi_{v} \simeq \widetilde{\Pi}_{v} \otimes \omega_{\pi_{v}}$, i.e. $\Pi$ is nearly equivalent to $\widetilde{\Pi} \otimes \omega$.

The representation $\Pi$ is equivalent to a subquotient of some representation

$$
\operatorname{Ind}\left(|\operatorname{det}|^{r_{1}} \sigma_{1} \otimes \cdots \otimes|\operatorname{det}|^{r_{t}} \sigma_{t}\right),
$$

where induction is from $\operatorname{GL}\left(n_{1}\right) \times \cdots \times \operatorname{GL}\left(n_{t}\right)$ with $n_{1}+\cdots+n_{t}=4$ to GL(4) and $\sigma_{i}$ are the unitary cuspidal automorphic representation of $\operatorname{GL}\left(n_{i}, \mathbb{A}\right)$ and $r_{i} \in \mathbb{R}$.

Without loss of generality we may assume that $r_{1} \geqslant r_{2} \geqslant \cdots \geqslant r_{t}$. Moreover, as $\Pi$ is unitary we have $n_{1} r_{1}+\cdots+n_{t} r_{t}=0$, which implies that $r_{t} \leqslant 0$. Let $T=S \cup\{v: v \mid \infty\}$ and consider

$$
L^{T}\left(s, \pi \times \widetilde{\sigma}_{t}\right)=L^{T}\left(s, \Pi \times \widetilde{\sigma}_{t}\right)=\prod_{i=1}^{t} L^{T}\left(s+r_{i}, \sigma_{i} \times \widetilde{\sigma}_{t}\right) .
$$

Here, $L^{T}$ denotes the product over $v \notin T$ of the local $L$-factors.

If $n_{t}=1$, then the left-hand side is entire by a result of Piatetski-Shapiro (cf. [Pia97, p. 274]). Now consider the right-hand side at $s_{0}=1-r_{t} \geqslant 1$. The last term in the product has a pole at $s_{0}$, whereas all of the others are non-zero there as $\Re\left(s_{0}+r_{i}\right)=1+r_{i}-r_{t} \geqslant 1$. This is a contradiction.

Now assume that $n_{t}=3$, i.e. $t=2$ with $n_{1}=1$ and $n_{2}=3$. Replacing $\pi$ and $\Pi$ by their contragredients will change $r_{i}$ to $-r_{i}$ and takes us back to the above situation, which gives a contradiction again.

Therefore, $n_{t}=2$. In this case, the left-hand side of (4) may have a pole at $s=1$ (cf. [PS84, Theorem 1.3] and the beginning of its proof), and if so, arguing as above, we conclude that $r_{t}=0$. This means that we either have $t=2$ with $n_{1}=n_{2}=2$ or $t=3$ with $n_{1}=n_{2}=1$ and $n_{2}=2$. However, we can rule out the latter as follows.

Assume that $t=3$ with $n_{1}=n_{2}=1$ and $n_{3}=2$. Then, it follows from the fact that $r_{3}=0$ and the conditions $r_{1} \geqslant r_{2} \geqslant r_{3}$ and $r_{1}+r_{2}+2 r_{3}=0$ that all of the $r_{i}$ would be zero in this case. This implies that if we consider the $L$-function of $\pi$ twisted by $\widetilde{\sigma}_{1}$, we have

$$
L^{T}\left(s, \pi \times \widetilde{\sigma}_{1}\right)=L^{T}\left(s, \sigma_{1} \times \widetilde{\sigma}_{1}\right) L^{T}\left(s, \sigma_{2} \times \widetilde{\sigma}_{1}\right) L^{T}\left(s, \sigma_{3} \times \widetilde{\sigma}_{1}\right) .
$$

Now the left-hand side is again entire by Piatetski-Shapiro's result mentioned above and the righthand side has a pole at $s=1$, which is a contradiction.

Therefore, the only possibilities are $t=1$ (i.e. $\Pi$ unitary cuspidal) or $t=2$ and $n_{1}=n_{2}=2$ with $r_{2}=0$. In the latter case, we also get $r_{1}=0$, as $r_{1}+r_{2}=0$ by unitarity of the central character. Moreover, in this case we have $\sigma_{1} \nsucceq \sigma_{2}$ as, otherwise,

$$
L^{T}\left(s, \pi \times \widetilde{\sigma}_{1}\right)=L^{T}\left(s, \sigma_{1} \times \widetilde{\sigma}_{1}\right) L^{T}\left(s, \sigma_{2} \times \widetilde{\sigma}_{1}\right)
$$

must have a double pole at $s=1$ while any possible pole of the left-hand side at $s=1$ is simple (cf. the proof of [PS84, Theorem 1.3]).

Therefore, we have proved the following.

Proposition 2.1. Let $\pi$ be a globally generic unitary cuspidal automorphic representation of $\operatorname{GSp}(4, \mathbb{A})$ and let $\Pi$ be any transfer of $\pi$ to $\mathrm{GL}(4, \mathbb{A})$. Then $\Pi$ is a subquotient of an automorphic representation as in (3) with either $t=1, n_{1}=4$ and $r_{1}=0$ (i.e. $\Pi$ is unitary cuspidal) or $t=2, n_{1}=n_{2}=2$ and $r_{1}=r_{2}=0$. In the latter case, we have $\sigma_{1} \not 7 \sigma_{2}$.

In fact, we can get more precise information. 


\section{Asgari And F. Shahidi}

Proposition 2.2. Let $\pi$ be a globally generic unitary cuspidal automorphic representation of $\operatorname{GSp}(4, \mathbb{A})$ with $\omega=\omega_{\pi}$ its central character and let $\Pi$ be any transfer as above. Then, $\Pi \simeq \widetilde{\Pi} \otimes \omega$ (not just nearly equivalent). Moreover:

(a) the representation $\Pi$ is cuspidal if and only if $\pi$ is not obtained as a Weil lifting from $\operatorname{GSO}(4, \mathbb{A})$;

(b) if $\Pi$ is not cuspidal, then it is the isobaric sum of two representations $\Pi=\Pi_{1} \boxplus \Pi_{2}$, where each $\Pi_{i}$ is a unitary cuspidal automorphic representation of $\operatorname{GL}(2, \mathbb{A})$ satisfying $\Pi_{i} \simeq \widetilde{\Pi}_{i} \otimes \omega$ and $\Pi_{1} \nsucceq \Pi_{2}$.

Proof. By Proposition 2.1, $\Pi$ is not cuspidal if and only if it is a subquotient of

$$
\Sigma=\operatorname{Ind}_{\mathrm{GL}(2, \mathbb{A}) \times \mathrm{GL}(2, \mathbb{A})}^{\mathrm{GL}(4, \mathbb{A})}\left(\sigma_{1} \otimes \sigma_{2}\right),
$$

where $\sigma_{i}$ are unitary cuspidal automorphic representation of $\operatorname{GL}(2, \mathbb{A})$.

On the other hand, by [PS84, Theorem 1.3] mentioned above, the representation $\pi$ is obtained as a Weil lifting from $\operatorname{GSO}(4, \mathbb{A})$ if and only if there exists an automorphic representation $\tau$ of $\mathrm{GL}(2, \mathbb{A})$ such that $L^{T}(s, \pi \times \tau)$ has a pole and in that case $\tau$ can be normalized so that the pole occurs at $s=1$.

Now assume that $\Pi$ is cuspidal. Then for any $\tau$ as above we have

$$
L^{T}(s, \pi \times \tau)=L^{T}(s, \Pi \times \tau),
$$

which is entire. Therefore, $\pi$ is not obtained as a Weil lifting from $\operatorname{GSO}(4, \mathbb{A})$. Moreover, as $\Pi$ is cuspidal, so is $\widetilde{\Pi} \otimes \omega$ and they are nearly equivalent, therefore, by the strong multiplicity one theorem [JS81a, JS81b, Pia79] we have $\Pi \simeq \widetilde{\Pi} \otimes \omega$.

Next, assume that $\Pi$ is not cuspidal and, hence, is given as a subquotient of $\Sigma$ above. We claim that each $\Sigma_{v}=\operatorname{Ind}\left(\sigma_{1, v} \otimes \sigma_{2, v}\right)$ is irreducible. To see this note that each $\sigma_{i, v}$ is generic unitary and is either a tempered representation of $\operatorname{GL}\left(2, k_{v}\right)$ or a complementary series $I\left(\chi||^{\alpha}, \chi||^{-\alpha}\right)$ with $\alpha \in\left(0, \frac{1}{2}\right)$ and $\chi$ a unitary character. If both of the $\sigma_{i, v}$ are tempered, then irreducibility of $\Sigma_{v}$ is clear. If both are complementary series of the form $I\left(\chi_{1}||^{\alpha}, \chi_{1}||^{-\alpha}\right)$ and $I\left(\chi_{2}||^{\beta}, \chi_{2}||^{-\beta}\right)$ with $\alpha, \beta \in\left(0, \frac{1}{2}\right)$ and $\chi_{i}$ unitary characters, then for $\Sigma_{v}$ to be reducible we should have $\alpha \pm \beta= \pm 1$, which is not possible. Finally, if one of $\sigma_{i, v}$ is tempered and the other is complementary series, then we have $\Sigma_{v}=$ $\operatorname{Ind}\left(\chi||^{-\alpha}, \chi_{1}, \chi_{2}, \chi||^{\alpha}\right), \Sigma_{v}=\operatorname{Ind}\left(\chi||^{-\alpha}, Q\left(\chi_{1}||^{-1 / 2}, \chi_{1}||^{1 / 2}\right), \chi||^{\alpha}\right)$ or $\Sigma_{v}=\operatorname{Ind}\left(\chi||^{-\alpha}, \eta, \chi||^{\alpha}\right)$. Here, $\chi_{1}, \chi_{2}$ and $\chi$ are unitary characters, $\alpha \in\left(0, \frac{1}{2}\right), \eta$ is a unitary supercuspidal representation of $\operatorname{GL}\left(2, k_{v}\right)$, and $Q\left(\chi_{1}||^{-1 / 2}, \chi_{1}||^{1 / 2}\right)$ denotes the Steinberg representation twisted by the unitary character $\chi_{1}$. Again, in all these cases the representation $\Sigma_{v}$ is irreducible as $\alpha \in\left(0, \frac{1}{2}\right)$.

Therefore, at every place $v$ the representation $\Pi_{v}$ is the same as the irreducible $\Sigma_{v}$ and, hence, it is the Langlands quotient. This means that $\Pi$ is an isobaric representation, i.e. the isobaric sum of $\sigma_{1}$ and $\sigma_{2}$. Again by the strong multiplicity one theorem, which remains valid for isobaric representations [JPS83], we conclude that $\Pi \simeq \widetilde{\Pi} \otimes \omega$. Now, just take $\Pi_{i}$ to be $\sigma_{i}$.

Finally, by [AS06, Proposition 7.4], which was based on classification theorems of Jacquet and Shalika, we know that we either have $\Pi_{i} \simeq \widetilde{\Pi}_{i} \otimes \omega$ for $i=1,2$ or we have $\Pi_{1} \simeq \widetilde{\Pi}_{2} \otimes \omega$ (or equivalently, $\left.\Pi_{2} \simeq \widetilde{\Pi}_{1} \otimes \omega\right)$. However, the latter case will not occur when $\pi$ is cuspidal and generic as, otherwise, $\pi$ will be nearly equivalent to an Eisenstein series representation, i.e. $\pi$ will be a CAP representation of $\operatorname{GSp}(4, \mathbb{A})$. This is impossible by [PS87, Theorem 1.1]. This completes the proof.

Remark 2.3. Note that any other transfer $\Pi^{\prime}$ of $\pi$ is also a subquotient of $\Sigma$ in (7), which is irreducible. Therefore, $\pi$ has a unique transfer to $\mathrm{GL}(4, \mathbb{A})$, which we continue to denote by $\Pi$. In particular, this implies that we have not lost any information at the places where we did not have a natural candidate for the local transfer.

Moreover, as $\Pi$ is either a unitary cuspidal representation of a general linear group or an isobaric sum of two such, every local representation $\Pi_{v}$ is full induced and generic. 


\section{Generic transfer From GSp(4) TO GL(4)}

Furthermore, if $\Pi$ is not cuspidal, then $\Pi_{1} \neq \Pi_{2}$ implies that $L\left(s, \Pi_{1} \times \widetilde{\Pi}_{2}\right)$ has no pole at $s=1$. This implies that the Fourier coefficient of $\Pi$ along the unipotent radical of our fixed Borel subgroup is non-vanishing, i.e. $\Pi$ is globally generic [Sha81].

We collect the above results in the following theorem, which is our main result.

TheOREM 2.4. Let $\pi$ be a globally generic unitary cuspidal automorphic representation of GSp $(4, \mathbb{A})$ with central character $\omega$. Then $\pi$ has a unique transfer $\Pi$ to $\operatorname{GL}(4, \mathbb{A})$, which satisfies $\Pi \simeq \widetilde{\Pi} \otimes \omega$ and its central character is $\omega^{2}$. Moreover, $\Pi$ is either unitary cuspidal or an isobaric sum $\Pi_{1} \boxplus \Pi_{2}$ of two inequivalent unitary cuspidal automorphic representations of $\operatorname{GL}(2, \mathbb{A})$ satisfying $\Pi_{i} \simeq \widetilde{\Pi}_{i} \otimes \omega$. The latter is the case if and only if $\pi$ is obtained as a Weil lifting from $\operatorname{GSO}(4, \mathbb{A})$. Furthermore, $\Pi$ is globally generic, i.e. it has a non-vanishing Fourier coefficient along the unipotent radical of our fixed Borel subgroup.

In fact, we can get more precise information about the local representations at places $v \in S$.

Proposition 2.5. Fix $v \in S$ and let

$$
\pi_{v} \simeq \operatorname{Ind}\left(\pi_{1, v}|\operatorname{det}|^{b_{1, v}} \otimes \cdots \otimes \pi_{t, v}|\operatorname{det}|^{b_{t, v}} \otimes \pi_{0, v}\right)
$$

be an irreducible generic representation of $\operatorname{GSp}\left(4, k_{v}\right)$, where each $\pi_{i, v}$ is a tempered representation of some $\mathrm{GL}\left(n_{i}, k_{v}\right), b_{1, v}>\cdots>b_{t, v}$, and $\pi_{0, v}$ is a tempered generic representation of some $\mathrm{GSp}\left(2 m, k_{v}\right)$. (Note that there are very few possibilities for $n_{i}$ and $m$ as $n_{1}+\cdots+n_{t}+m=2$. We are allowing $m=0$ and, by convention, $\mathrm{GSp}(0)=\mathrm{GL}(1)$.) Let $\omega_{v}$ denote the central character of $\pi_{v}$. Assume that $\pi_{v}$ is the local component of the globally generic unitary cuspidal representation $\pi$ of $G \operatorname{Sp}(4, \mathbb{A})$ and let $\Pi$ be its transfer to $\mathrm{GL}(4, \mathbb{A})$. Then the local component $\Pi_{v}$ of $\Pi$ at $v$ is generic and of the form

$$
\Pi_{v} \simeq \operatorname{Ind}\left(\pi_{1, v}|\operatorname{det}|^{b_{1, v}} \otimes \cdots \otimes \pi_{t, v}|\operatorname{det}|^{b_{t, v}} \otimes \Pi_{0, v} \otimes\left(\widetilde{\pi}_{t, v} \otimes \omega_{v}\right)|\operatorname{det}|^{-b_{t, v}} \otimes \cdots \otimes\left(\widetilde{\pi}_{1, v} \otimes \omega_{v}\right)|\operatorname{det}|^{-b_{1, v}}\right),
$$

where $\Pi_{0, v}$ is a tempered generic representation of $\mathrm{GL}\left(2 m, k_{v}\right)$ if $m>0$.

Proof. Let us remark that, as in [CKPS04, §7], one could define the notion of local transfer and obtain complete information about such transfers for a general irreducible admissible generic representation, whether a local component of a global representation or not. (In fact, the representation $\Pi_{0, v}$ would then be the local transfer of $\pi_{0, v}$. $)$ However, we do not need the full extent of such results in this paper.

Recall that we already proved (cf. Remark 2.3) that each $\Pi_{v}$ is generic and is full induced. Let $v \in S$ and consider $\pi_{v}$ and $\Pi_{v}$ as in the proposition. We first show that if $\rho_{v}$ is any supercuspidal representation of $\mathrm{GL}\left(r, k_{v}\right)$, then

$$
\gamma\left(s, \pi_{v} \times \rho_{v}, \psi_{v}\right)=\gamma\left(s, \Pi_{v} \times \rho_{v}, \psi_{v}\right) .
$$

The key here is the fact that there exists a unitary cuspidal representation $\rho$ of $\mathrm{GL}(r, \mathbb{A})$ such that its local component at $v$ is $\rho_{v}$ and at all other finite places $w \neq v$ the local component $\rho_{w}$ is unramified (cf. [Sha90, Proposition 5.1]). Now applying the converse theorem of Cogdell and Piatetski-Shapiro with $S^{\prime}=S-\{v\}$ will give the result exactly as in the proof of [CKPS04, Proposition 7.2]. Moreover, by multiplicativity of $\gamma$-factors, we conclude that (10) also holds if $\rho_{v}$ is a discrete series representation of $\mathrm{GL}\left(r, k_{v}\right)$.

Next, we claim that if $\pi_{v}$ is tempered, then so is $\Pi_{v}$. Here, again the main tool is multiplicativity of $\gamma$-factors and the proof is exactly as in [CKPS04, Lemma 7.1]. This proves the proposition for the case $m=2$. If $m=1$, then the group $\operatorname{GSp}(2 m)=\operatorname{GSp}(2)$ is the same as $\operatorname{GL}(2)$ and we set $\Pi_{0, v}$ to be $\pi_{0, v}$ itself. For $m=0$ we need no choice of $\Pi_{0, v}$. Now, let $T=\left\{w_{0}\right\}$ consist of a single finite place $w_{0} \neq v$ at which $\pi_{v}$ is unramified and consider the representation $\Pi^{\prime}$ of $\operatorname{GL}(4, \mathbb{A})$ whose local 


\section{Asgari And F. Shahidi}

components are the same as $\Pi$ outside of $S$ and are the irreducible induced representations on the right-hand side of (9) when $v \in S$. We can now apply the converse theorem again to $\Pi^{\prime}$ and $T=\left\{w_{0}\right\}$ to conclude that $\Pi^{\prime}$ is a transfer of $\pi$. The key here is that the induced representations on the right-hand side of (9) have the right $L$-functions. Therefore, by uniqueness of the transfer we proved earlier, we have $\Pi_{v}^{\prime} \simeq \Pi_{v}$ for $v \in S$. This completes the proof.

\section{Applications}

We first recall that the current formulation of the Ramanujan conjecture for generic cuspidal representations states that for any quasi-split group and any globally generic unitary cuspidal automorphic representation $\pi=\bigotimes_{v} \pi_{v}$, the local components $\pi_{v}$ are tempered for all places $v$. As an application of our main theorem, we can prove two types of results in this direction: estimates toward this conjecture for the group GSp(4) as well as a weaker version of it for generic representations of this group.

\subsection{Estimates toward Ramanujan}

Following [CKPS04], we introduce the following notation in order to prove estimates. Let $\Pi=\bigotimes_{v} \Pi_{v}$ be a unitary cuspidal automorphic representation of $\operatorname{GL}\left(m, \mathbb{A}_{k}\right)$. For every place $v$ the representation $\Pi_{v}$ is unitary generic and can be written as a full induced representation

$$
\Pi_{v} \simeq \operatorname{Ind}\left(\Pi_{1, v}|\operatorname{det}|^{a_{1, v}} \otimes \cdots \otimes \Pi_{t, v}|\operatorname{det}|^{a_{t, v}}\right)
$$

with $a_{1, v}>\cdots>a_{t, v}$ and each $\Pi_{i, v}$ tempered [Vog78, Zel80].

Definition 3.1. We say $\Pi$ satisfies $H\left(\theta_{m}\right)$ with $\theta_{m} \geqslant 0$ if for all places $v$ we have $-\theta_{m} \leqslant a_{i, v} \leqslant \theta_{m}$.

The classification of generic unitary dual of $\mathrm{GL}(m)$ (see [Tad86, Vog78]) trivially gives $H\left(\frac{1}{2}\right)$. The best result currently known for a general number field $k$ says that any unitary cuspidal representation of $\operatorname{GL}(m, \mathbb{A})$ satisfies $H\left(\frac{1}{2}-1 /\left(m^{2}+1\right)\right)$ (see [LRS99]). When $k=\mathbb{Q}$ and $m \leqslant 4$, it is $H\left(\frac{1}{2}-1 /(1+m(m+1) / 2)\right)$. The same bound is also available for $m>4$ for $k=\mathbb{Q}$ provided that one knows that the symmetric square $L$-function of $\Pi$ is absolutely convergent for $\Re(s)>1$ (cf. [KS03]), but this is only currently available for $m \leqslant 4$. When $m=2$ we have the better bounds of $H\left(\frac{1}{9}\right)$ for a general number field $k$ (see $[\mathrm{KS} 02]$ ) and $H\left(\frac{7}{64}\right)$ for $k=\mathbb{Q}$ (see [KS03]). The Ramanujan conjecture demands $H(0)$.

Similarly, if $\pi=\bigotimes_{v} \pi_{v}$ is a unitary generic cuspidal automorphic representation of $\operatorname{GSp}\left(2 n, \mathbb{A}_{k}\right)$, then by [Mui01] and [Vog78], each $\pi_{v}$ can be written as a full induced representation

$$
\pi_{v} \simeq \operatorname{Ind}\left(\pi_{1, v}|\operatorname{det}|^{b_{1, v}} \otimes \cdots \otimes \pi_{t, v}|\operatorname{det}|^{b_{t, v}} \otimes \tau_{v}\right),
$$

where each $\pi_{i, v}$ is a tempered representation of some $\operatorname{GL}\left(n_{i}, k_{v}\right)$ and $\tau_{v}$ is a tempered generic representation of some $\operatorname{GSp}\left(2 m, k_{v}\right)$ with $n_{1}+\cdots+n_{t}+m=n$.

Definition 3.2. We say $\pi$ satisfies $H\left(\theta_{n}\right)$ with $\theta_{n} \geqslant 0$ if for all places $v$ we have $-\theta_{n} \leqslant b_{i, v} \leqslant \theta_{n}$.

The classification of generic unitary dual of GSp(4) (cf. [LMT04], for example) trivially gives the estimate $H(1)$. The Ramanujan conjecture demands $H(0)$ again. For a survey of results in this direction and their applications we refer to [Sar03, Sha04].

THEOREM 3.3. Let $k$ be a number field and assume that all unitary cuspidal representations of $\operatorname{GL}\left(4, \mathbb{A}_{k}\right)$ (respectively, $\operatorname{GL}\left(2, \mathbb{A}_{k}\right)$ ) satisfy $H\left(\theta_{4}\right)$ (respectively, $H\left(\theta_{2}\right)$ ) and $\theta_{2} \leqslant \theta_{4}$. Then any globally generic unitary cuspidal representation $\pi$ of $\operatorname{GSp}\left(4, \mathbb{A}_{k}\right)$ satisfies $H\left(\theta_{4}\right)$, and even the better bound of $H\left(\theta_{2}\right)$ if $\pi$ transfers to a non-cuspidal representation of $\mathrm{GL}\left(4, \mathbb{A}_{k}\right)$ (cf. Theorem 2.4). 


\section{Generic transfer from GSp(4) To GL(4)}

Proof. Let $\Pi$ be the functorial transfer of $\pi$ to $\mathrm{GL}\left(4, \mathbb{A}_{k}\right)$. If $v$ is an archimedean place of $k$, then the theorem is clear as in this case local functoriality is well understood through Langlands' parametrization (cf. the proof of [AS06, Theorem 6.1], for example, for more details).

Let $v$ be a non-archimedean place of $k$ at which $\pi_{v}$ is unramified. Then it follows from (2) that $\pi_{v}$ is given by its Frobenius-Hecke (Satake) parameter, which is of the form

$$
\operatorname{diag}\left(\chi_{1}(\varpi), \chi_{2}(\varpi), \chi_{2}^{-1}(\varpi) \chi_{0}(\varpi), \chi_{1}^{-1}(\varpi) \chi_{0}(\varpi)\right),
$$

where $\varpi$ denotes a uniformizer of $k_{v}$. If $\Pi$ is cuspidal, then for $i=1,2$ we have by assumption

$$
q_{v}^{-\theta_{4}} \leqslant\left|\chi_{i}(\varpi)\right| \leqslant q_{v}^{\theta_{4}} .
$$

If $\Pi$ is not cuspidal, then we have similar inequalities with $\theta_{4}$ replaced by the even better estimate of $\theta_{2}$. As $\pi_{v}$ is unitary, we have $\left|\chi_{0}(\varpi)\right|=1$. Therefore, Frobenius-Hecke parameters of $\pi_{v}$ also satisfy similar inequalities.

Next, assume that $v$ is a place of $k$ in $S$. Then, by Proposition 2.5, a similar argument as above works again.

Corollary 3.4. Let $\pi$ be a globally generic unitary cuspidal representation of $\operatorname{GSp}\left(4, \mathbb{A}_{k}\right)$. Then $\pi$ satisfies $H\left(\frac{15}{34}\right)$. If $\pi$ transfers to a non-cuspidal representation of $\mathrm{GL}\left(4, \mathbb{A}_{k}\right)$, then it satisfies $H\left(\frac{1}{9}\right)$. If $k=\mathbb{Q}$, then we have the better estimates of $H\left(\frac{9}{22}\right)$ and $H\left(\frac{7}{64}\right)$, respectively.

Proof. The proof is immediate if we combine Theorem 3.3 with the known results on estimates for the general linear groups mentioned above.

COROLlary 3.5. The Ramanujan conjecture for unitary cuspidal representations of GL(4) and GL(2) imply the Ramanujan conjecture for the generic spectrum of GSp(4).

\subsection{The weak Ramanujan conjecture}

Following [CP95, Ram97, Kim03] we recall the following definition.

Definition 3.6. Let $\mathbf{G}$ be a split reductive group over the number field $k$. Let $\pi=\bigotimes \pi_{v}$ be an automorphic representation of $\mathbf{G}\left(\mathbb{A}_{k}\right)$. We say that $\pi$ is weakly Ramanujan if, given $\epsilon>0$, there exists a set $T$ of places of $k$ containing the archimedean places and the non-archimedean ones with $\pi_{v}$ ramified such that $T$ has density zero and for $v \notin T$ the Frobenius-Hecke parameter $\operatorname{diag}\left(\lambda_{v, i}\right)$ of $\pi_{v}$ satisfies

$$
\max _{i}\left\{\left|\lambda_{v, i}\right|,\left|\lambda_{v, i}^{-1}\right|\right\} \leqslant q_{v}^{\epsilon}
$$

Here, $q_{v}$ denotes the cardinality of the residue field.

We are concerned with the cases of $\mathbf{G}=\mathrm{GL}(m)$ or $\mathbf{G}=\mathrm{GSp}(4)$ in this paper. We recall that (unitary) cuspidal representations of $\mathrm{GL}(m)$ for $m \leqslant 4$ are weakly Ramanujan (cf. [Ram97] and [Kim03, Propositions 3.7 and 6.3]).

Let $\pi$ be a globally generic unitary cuspidal representation of $\operatorname{GSp}\left(4, \mathbb{A}_{k}\right)$. For any $v \notin T$ as above, let

$$
\operatorname{diag}\left(a_{0, v}, a_{1, v}, a_{2, v}\right)
$$

be the Frobenius-Hecke parameter of $\pi_{v}$ (cf. (1)). Then, as in (13), the parameter of the local transfer $\Pi_{v}$ is given by

$$
\operatorname{diag}\left(a_{1, v}, a_{2, v}, a_{2, v}^{-1} a_{0, v}, a_{1, v}^{-1} a_{0, v}\right) .
$$




\section{Asgari And F. Shahidi}

Moreover, $\left|a_{0, v}\right|=1$ as $\pi_{v}$ is unitary. Therefore, the above results about weak Ramanujan property of unitary cuspidal representations of $\mathrm{GL}(m)$ immediately imply the following.

TheOREM 3.7. Let $\pi$ be a globally generic unitary cuspidal representation of $\operatorname{GSp}\left(4, \mathbb{A}_{k}\right)$. Then $\pi$ is weakly Ramanujan.

\subsection{Spinor $L$-function for $\operatorname{GSp}(4)$}

As another application we get the following immediate corollary of our main result, Theorem 2.4.

Proposition 3.8. Let $\pi$ be a globally generic unitary cuspidal representation of $\operatorname{GSp}\left(4, \mathbb{A}_{k}\right)$. Then the spinor $L$-function $L(s, \pi$, spin $)$ is entire.

Proof. Let $\Pi$ be the transfer of $\pi$ to $\mathrm{GL}(4, \mathbb{A})$. If $\Pi$ is unitary cuspidal, then $L(s, \pi, \operatorname{spin})=L(s, \Pi)$ and if $\Pi=\Pi_{1} \boxplus \Pi_{2}$ is the isobaric sum of two unitary cuspidal representations of GL $(2, \mathbb{A})$, then we have $L(s, \pi, \operatorname{spin})=L\left(s, \Pi_{1}\right) L\left(s, \Pi_{2}\right)$. In either case the $L$-functions on the right-hand side are standard $L$-functions of unitary cuspidal representations of the general linear group and are entire.

Remark 3.9. This result has also been proved for a certain class of globally generic representations $\pi$ by Takloo-Bighash in [Tak]. His methods are different from ours and are based on using an integral representation. T. Moriyama has proved certain cases of this result as well [Mor04, IM05].

\section{ACKNOWLEDGEMENTS}

We would like to thank James Cogdell for many helpful discussions and Peter Sarnak for his interest in this work. The first author would also like to thank Steve Gelbart, Dihua Jiang, Robert Langlands, Brooks Roberts and Ramin Takloo-Bighash for many helpful discussions during the course of this work.

\section{REFERENCES}

Art04 J. Arthur, Automorphic representations of GSp(4), in Contributions to automorphic forms, geometry, and number theory (Shalika volume), eds H. Hida, D. Ramakrishnan and F. Shahidi (Johns Hopkins University Press, Baltimore, MD, 2004), 65-81.

AS06 M. Asgari and F. Shahidi, Generic transfer for general spin groups, Duke Math. J. 132 (2006), 137-190.

CKPS04 J. Cogdell, H. Kim, I. Piatetski-Shapiro and F. Shahidi, Functoriality for the classical groups, Publ. Math. Inst. Hautes Études Sci. 99 (2004), 163-233.

CP95 J. Cogdell and I. Piatetski-Shapiro, Unitarity and functoriality, Geom. Funct. Anal. 5 (1995), 164-173.

GRS01 D. Ginzburg, S. Rallis and D. Soudry, Generic automorphic forms on $\mathrm{SO}(2 n+1)$ : functorial lift to GL(2n), endoscopy, and base change, Internat. Math. Res. Notices 14 (2001), 729-764.

IM05 T. Ishi and T. Moriyama, Spinor L-functions for generic cusp forms on GSp(2) belonging to principal series representations, Preprint (2005).

JPS83 H. Jacquet, I. Piatetskii-Shapiro and J. Shalika, Rankin-Selberg convolutions, Amer. J. Math. 105 (1983), 367-464.

JS81a H. Jacquet and J. Shalika, On Euler products and the classification of automorphic representations. I, Amer. J. Math. 103 (1981), 499-558.

JS81b H. Jacquet and J. Shalika, On Euler products and the classification of automorphic forms. II, Amer. J. Math. 103 (1981), 777-815.

Kim01 H. Kim, Residual spectrum of odd orthogonal groups, Internat. Math. Res. Notices 17 (2001), 873-906. 


\section{Generic transfer from GSp(4) To GL(4)}

Kim02 H. Kim, Applications of Langlands' functorial lift of odd orthogonal groups, Trans. Amer. Math. Soc. 354 (2002), 2775-2796.

Kim03 H. Kim, Functoriality for the exterior square of $\mathrm{GL}_{4}$ and the symmetric fourth of $\mathrm{GL}_{2}$ (with Appendix 1 by D. Ramakrishnan and Appendix 2 by H. Kim and P. Sarnak), J. Amer. Math. Soc. 16 (2003), 139-183.

KS02 H. Kim and F. Shahidi, Cuspidality of symmetric powers with applications, Duke Math. J. 112 (2002), 177-197.

KS03 H. Kim and P. Sarnak, Refined estimates towards the Ramanujan and Selberg conjectures, J. Amer. Math. Soc. 16 (2003). Appendix 2 to [Kim03].

Lan89 R. Langlands, On the classification of irreducible representations of real algebraic groups, in Representation theory and harmonic analysis on semisimple Lie groups, Mathematical Surveys and Monographs, vol. 31 (American Mathematical Society, Providence, RI, 1989), 101-170.

LMT04 E. Lapid, G. Muić and M. Tadić, On the generic unitary dual of quasisplit classical groups, Internat. Math. Res. Notices 26 (2004), 1335-1354.

LRS99 W. Luo, Z. Rudnick and P. Sarnak, On the generalized Ramanujan conjecture for GL( $n)$, in Automorphic forms, automorphic representations, and arithmetic, Fort Worth, TX, 1996, Proceedings of Symposia in Pure Mathematics, vol. 66 (American Mathematical Society, Providence, RI, 1999), 301-310.

Mor04 T. Moriyama, Entireness of the spinor L-functions for certain generic cusp forms on GSp(2), Amer. J. Math. 126 (2004), 899-920.

Mui01 G. Muić, A proof of Casselman-Shahidi's conjecture for quasi-split classical groups, Canad. Math. Bull. 44 (2001), 298-312.

Pia79 I. Piatetski-Shapiro, Multiplicity one theorems, in Automorphic forms, representations and L-functions, Oregon State University, Corvallis, OR, 1977, part 1, Proceedings of Symposia in Pure Mathematics, vol. 33 (American Mathematical Society, Providence, RI, 1979), 209-212.

Pia97 I. Piatetski-Shapiro, L-functions for GSp 4 . Olga Taussky-Todd: in memoriam, Pacific J. Math. 181 Special Issue (1997), 259-275.

PS84 I. Piatetski-Shapiro and D. Soudry, $L$ and $\varepsilon$ functions for GSp(4) $\times$ GL(2), Proc. Natl Acad. Sci. USA 81 (1984), 3924-3927.

PS87 I. Piatetski-Shapiro and D. Soudry, On a correspondence of automorphic forms on orthogonal groups of order five, J. Math. Pures Appl. (9) 66 (1987), 407-436.

Ram97 D. Ramakrishnan, On the coefficients of cusp forms, Math. Res. Lett. 4 (1997), 295-307.

Sar03 P. Sarnak, Notes on the generalized Ramanujan conjectures, Fields Institute Lectures, 2003, in Harmonic analysis, the trace formula, and Shimura varieties, Clay Mathematics Proceedings, vol. 4 (American Mathematical Society, Providence, RI, 2005), 659-685.

Sha81 F. Shahidi, On certain L-functions, Amer. J. Math. 103 (1981), 297-355.

Sha90 F. Shahidi, A proof of Langlands' conjecture on Plancherel measures; complementary series for p-adic groups, Ann. of Math. (2) 132 (1990), 273-330.

Sha04 F. Shahidi, On the Ramanujan conjecture for quasi-split groups, Asian J. Math. 8 (2004), 813-836.

Sou05 D. Soudry, On Langlands functoriality from classical groups to $\mathrm{GL}_{n}$, Astérisque 298 (2005), 335-390.

Tad86 M. Tadić, Classification of unitary representations in irreducible representations of general linear group (non-Archimedean case), Ann. Sci. École Norm. Sup. (4) 19 (1986), 335-382.

Tak R. Takloo-Bighash, Spinor L-functions, theta correspondence, and Bessel coefficients, Forum Math., to appear.

Vog78 D. Vogan, Gelfand-Kirillov dimension for Harish-Chandra modules, Invent. Math. 48 (1978), 75-98.

Zel80 A. Zelevinsky, Induced representations of reductive p-adic groups II. On irreducible representations of GL(n), Ann. Sci. École Norm. Sup. (4) 13 (1980), 165-210. 


\section{Generic transfer from GSp(4) To GL(4)}

Mahdi Asgari asgari@math.okstate.edu

School of Mathematics, Institute for Advanced Study, Princeton, NJ 08450, USA

Current address: Department of Mathematics, Oklahoma State University, Stillwater, OK 74078-1058, USA

Freydoon Shahidi shahidi@math.purdue.edu

Department of Mathematics, Purdue University, West Lafayette, IN 47907, USA 